\title{
Self-organization of clusters by a standing surface acoustic wave
}

\author{
Christophe Taillan, Nicolas Combe, and Joseph Morillo \\ Centre d'Elaboration de Matériaux et d'Etudes Structurales, CNRS UPR 8011, 29 rue J. Marvig, BP 94347, 31055 Toulouse cedex 4, France \\ and Université de Toulouse; UPS; F-31055 Toulouse, France
}

(Received 17 March 2017; published 12 July 2017)

\begin{abstract}
The diffusion of clusters on a crystalline substrate submitted to a standing surface acoustic wave (StSAW) is studied using molecular dynamics simulations. The distributions of positions of clusters with two, three, and four atoms are calculated and evidence that the wave encourages the presence of the clusters in the vicinity of the maximum transverse displacement field of the substrate. The physical mechanism leading to this self-organization is expected to be equivalent to the one operating for a single adatom, i.e., the displacement of the clusters induced by the longitudinal displacement field of the wave. The detailed shapes of the distributions of positions of clusters are related to the different clusters' orientation and configurations. Finally, the possibility to use a StSAW to self-organize nanostructures during growth is addressed by simulating a deposition process on the substrate. We evidence that the use of a StSAW allows to especially control the spatial repartition of grown nanostructures.
\end{abstract}

DOI: 10.1103/PhysRevB.96.035414

\section{INTRODUCTION}

Lithography, though its high cost and time-consuming stages, remains the current most used and reliable technique to produce fashioned nanostructures. Besides, self-organization techniques allow a quick and cheap way to produce arrangement of nanostructures. However, the control of their sizes, repartition, and spacing is usually difficult and not reliable, preventing the use of self-organization techniques in industrial processes. Hence, improvement of this control remains both a challenging task and an active research field. The most known self-organization techniques are the Stranski-Krastanov growth mode [1,2], the use of buried dislocation networks [3] of nanopatterned substrates [4] or of diblock copolymers [5].

Recently, we have proposed to use a standing acoustic wave in order to self-organize the adatom diffusion on a crystalline substrate. Analogously to the self-organization of bunches of sand deposited on a vibrating metallic plate forming Chladni figures, the diffusion of adatoms can be driven by a surface standing acoustic wave (StSAW) propagating in the substrate. The operation of this self-organization has been evidenced using molecular dynamics (MD) simulations [6] by showing that the probability to find an adatom in the vicinity of an antinode of the transversal substrate displacement is enhanced. The physical mechanism inducing this self-organization has been revealed by investigating the interaction between the adatom and the substrate [7-9]. The crystalline substrate induces on the adatom a spatially periodic potential of which minima are periodically (in time) displaced by the longitudinal displacement field of the wave. Such minima displacement induces a force on the adatom, responsible for the self-organization.

While we have focused on statistic properties, other groups have investigated kinetic aspects: theoretically and experimentally studying the diffusion of adatoms or clusters on a crystalline substrate submitted to a propagative SAW, a strong enhancement of the surface diffusion has been observed and explained by a mechanism based on the modulation of both the diffusion energy barriers and of the local temperature by the SAW [10,11].

Following our previous approach focusing on the probability density of presence of a diffusing adatom, this paper considers the effect of the StSAW on the probability density of small clusters. This effect is investigated using MD simulations, details of which are described in Sec. II. In Sec. III, we report, explain, and discuss our main results evidencing the self-organization of a cluster of two, three, and four atoms on a crystalline substrate induced by a StSAW. Finally, in Sec. IV, we show that the StSAW can self-organize the growth of nanostructures on a substrate, evidencing the effect of StSAW on few tens of atom clusters. Section V concludes this work and proposes various future researches directions.

\section{SIMULATION DETAILS}

The diffusion of clusters on a (001) fcc surface submitted to a StSAW propagating in the [110] direction is considered. MD simulations are performed using the LAMMPS simulation package [12]. The substrate is modeled by a fcc crystal slab. A sketch of the simulation cell is reported in Fig. 1. Periodic boundary conditions are applied in the $x$ and $y$ directions corresponding to the [110] and [110] crystal directions. The upper slab surface is perpendicular to the $z$ direction corresponding to the [001] direction. The StSAW is created by imposing a periodic displacement of amplitude $A$ along the $z$ direction to a small group of atoms closed to the surface: these atoms, displayed in a dashed region in Fig. 1, belong to the three top layers of the substrate and to three adjacent $x$ planes. This motion creates two counterpropagating Rayleigh waves of wavelength $\lambda$ propagating along the [110] direction: the angular frequency $\Omega$ of the excitation is adjusted so that the cell $x$ size $L_{x}$ is a multiple of the wavelength (in this work $L_{x}=2 \lambda$ ), warranting that the two waves form a StSAW. In addition, in order to fully decorrelate the effect of the StSAW from the substrate crystalline structure, the StSAW wavelength is chosen large compared to the crystal lattice parameter $a_{x}$ in the $x$ direction: $\lambda=17 a_{x}$. The $y$ size $L_{y}$ of the substrate is chosen from the cutoff distance of the interaction potential in order to prevent any interaction of any atom with its own $y$-periodic image. The width of the slab is chosen of the order of the characteristic attenuation length of the StSAW in the substrate, i.e., roughly the wavelength $\lambda$. The substrate is 


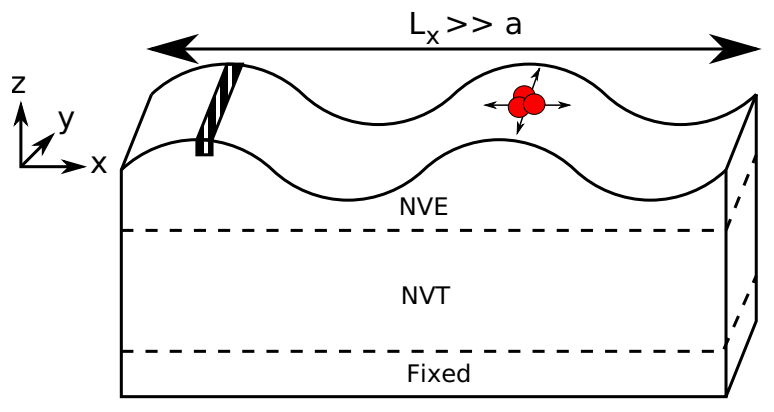

FIG. 1. Sketch of the simulation model. The cluster (formed of red atoms) diffuses on the upper crystal surface. Atoms in the dashed region have an imposed vertical sinusoidal displacement (frequency $\Omega$ ) and produce a StSAW along the $x$ direction. The atoms of the 12 upper layers of the substrate are in the microcanonical ensemble (NVE). The atoms in the large intermediate region (15 layers) are thermostatted (NVT ensemble) and the 4 bottom layers of the substrate are kept fixed.

composed of 31 (001) planes. The 4 bottom ones are fixed in order to prevent any overall motion of the slab in the $z$ direction: this constraint intends to simulate a semi-infinite crystal in the $-z$ direction. Since the imposed displacement to atoms used to create the StSAW injects some energy in the system, a NVT Nose-Hoover thermostat is applied to the substrate to absorb this energy and warrants a constant temperature in the system. However, in order to prevent any attenuation of StSAW along the $x$ direction and interaction of the thermostat with the diffusing clusters, the 12 top layers of the substrate as well as the atoms in the clusters are in the microcanonical ensemble and are thermalized by the underlying crystal.

Since the self-organization by a StSAW has been shown to be independent on the precise choice of the semiempirical potential [6], a computationally cheap Lenard-Jones pair potential is chosen for this study. The interaction pair potential between atoms $\alpha$ and $\beta$ is

$$
V_{\alpha \beta}(r)=4 \epsilon_{\alpha \beta}\left[\left(\frac{\sigma_{\alpha \beta}}{r}\right)^{12}-\left(\frac{\sigma_{\alpha \beta}}{r}\right)^{6}\right],
$$

where $(\alpha, \beta) \in\{s, c\}$, where $s$ and $c$ design substrate and cluster atoms. Interaction parameters for the substrate atoms are $\epsilon_{s s}=1, \sigma_{s s}=1$, and $m_{s}=1$ (substrate atom mass). In the following, times, distances, masses, energy, and temperatures are, respectively, expressed in units of $\sqrt{\frac{m_{s} \sigma_{s s}^{2}}{\epsilon_{s s}}}, \sigma_{s s}, m_{s}, \epsilon_{s s}$, and $\frac{\epsilon_{s s}}{k_{b}}\left(k_{B}\right.$ the Boltzmann constant). A 2.63 cutoff distance with a polynomial switching function is applied to smoothly truncate the Lennard-Jones potential.

Interaction parameters for cluster-cluster and clustersubstrate interactions are $\epsilon_{c c}=2, \sigma_{c c}=1$, and $m_{c}=1$ (cluster atom mass) and $\epsilon_{s c}=0.82, \sigma_{s c}=1$. Within this choice, evaporation of atoms, exchange of cluster atoms with substrate atoms [9], or dissociation of clusters are very scarce on the simulation time scale. However, rigorously, in absence of a two-dimensional (2D) adatom gas on the substrate surface at the saturated pressure, the studied clusters are not thermodynamically stable against dissociation. The amplitude of the StSAW is chosen in order to prevent any plastic deformation of the substrate and is fixed by the amplitude of the atom group displacement $A=0.4$. The substrate temperature is fixed to $T=0.24$. This temperature has been optimized in order to allow a significant diffusion of the clusters on affordable simulation time scales. Increasing this temperature would result in an increase of the rate of unwanted processes (noticeably the dissociation of clusters), while decreasing this temperature will slow down the diffusion and require a longer simulation time scale to get good statistical averages. Within this choice of parameters, the simulations using the Lennard-Jones potential roughly correspond to the ones of a noble metal at about $1000 \mathrm{~K}$ [13].

\section{SELF-ORGANIZATION OF SMALL CLUSTERS}

Atoms forming clusters are deposited on the substrate surface submitted to the StSAW. The trajectories of the center of mass of the clusters are studied. A statistical analysis is performed from 200 trajectories of 8000 time units, with evenly spaced initial cluster positions on a StSAW wavelength. The conformations of the clusters varying along the trajectory, and the simulation results do not depend on the initial conformations of the clusters. The probability distributions $p_{i}(x, y)$ of the $x, y$ coordinates of centers of mass of clusters composed of $i$ atoms, $i \in\{1,2,3,4\}$, are computed. Figures 2(a)-2(d) report the normalized probability distributions $P_{i}(x)=\int_{0}^{L_{y}} p_{i}(x, y) d y$ of the $x$ coordinate of these centers of mass. Results of Fig. 2(a) have already been reported in Ref. [9] but are shown here as a reference. Figure 2(e) reports the transverse substrate displacement field $z(x)$ of the top substrate atomic layer at 2 times corresponding to antinode maximum displacements. All investigated probability distributions $P_{i}(x), i \in\{1,2,3,4\}$, display an oscillation induced by the crystalline potential (on a length scale of the order of the substrate lattice parameter) whose maxima are spatially modulated at half the StSAW wavelength. The periodicity of these oscillations is discussed in detail below in Sec. III A. Figure 2 clearly shows that the probability that an adatom or a small cluster is in the vicinity of an antinode of the transverse displacement field is enhanced. This latter result constitutes the main conclusion of this work: clusters also self-organize in the presence of a StSAW. This self-organization will be quantified and discussed in Sec. III B.

\section{A. Probability distribution}

The main differences between adatom and cluster diffusion are (i) the characteristic diffusion time which increases with the cluster size and (ii) the different geometric conformations that the clusters can adopt. Point (i) has not been investigated in this study since it has been addressed by various authors [14-19]. The point (ii) is examined here through the statistical analysis of the cluster trajectories: the different geometric cluster conformations are responsible of the position of the oscillation maxima reported in Fig. 2.

For a single diffusing adatom, $P_{1}(x)$ displays oscillations with a $a_{x}$ period: the adatom spends a significant time in the vicinity of minima of the crystalline potential. These minima are spaced every $a_{x}$ along the [110] direction corresponding to the periodicity of the maxima of $P_{1}(x)$ : the probability 

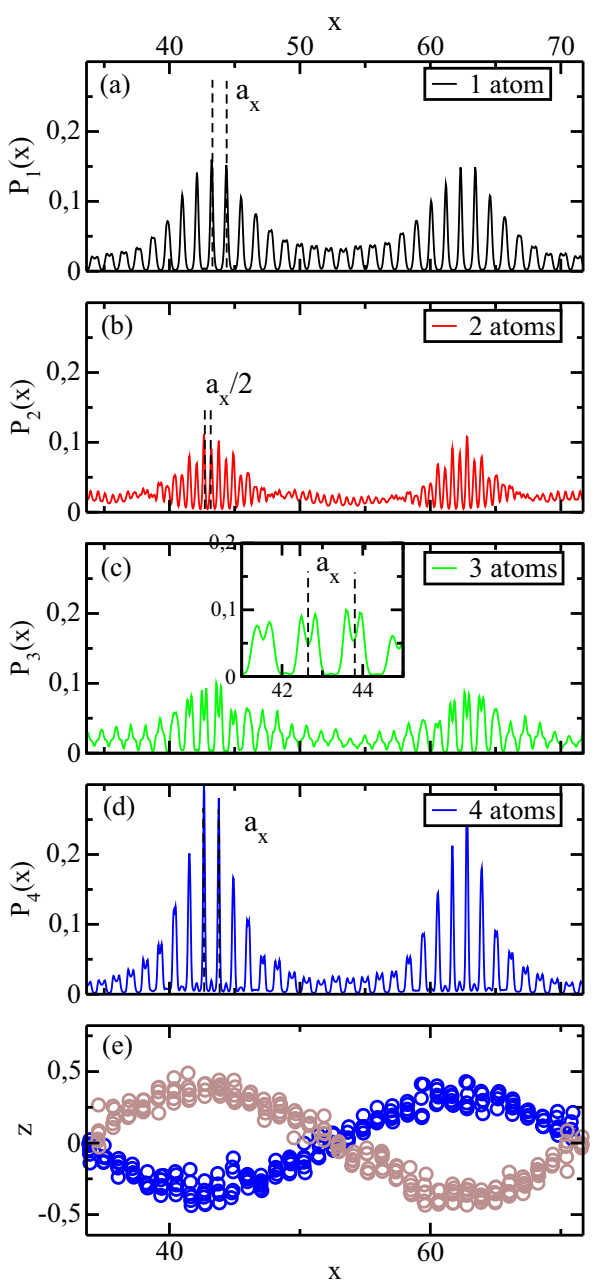

FIG. 2. (a)-(d) Probability distributions $P_{i}(x)$ of the $x$ coordinate of centers of mass of clusters composed of $i$ atoms, $i \in\{1,2,3,4\}$. The distributions are obtained from $200 \mathrm{MD}$ trajectories of 8000 time units at $T=0.24$ in the presence of a StSAW propagating along the [110] direction and with amplitude $A=0.4$ and wavelength $\lambda=38.08$. The inset of Fig. 2(c) is a zoom-in the region $x \in[41,45]$ evidencing the double peak character of each maxima. (e) Displacement $z(x)$ of the top substrate atomic layer at 2 times corresponding to antinode maximum displacements.

distribution $P_{1}(x)$ has already been discussed elsewhere [9]. We focus below on the probability distributions $P_{i}(x)$ with $i \in\{2,3,4\}$. Figure 3 reports the main conformations of the clusters of two, three, and four atoms on the (001) surface. For two-atom clusters, the two conformations referred as $i_{21}$ and $i_{22}$ correspond to the stable states of the cluster on the surface. For three- or four-atom clusters, both stable (referred as $i_{3}$ and $i_{4}$ ) or metastable (referred as $m_{i j}$ with $i \in\{3,4\}$ and $j \in\{1,2,3,4\}$ ) conformations of the clusters are reported. Here, the stability or metastability is defined from the conformation energies calculated at $0 \mathrm{~K}$ in absence of any StSAW. The energy of a cluster is here calculated as the difference between the energies of the cluster on the substrate and of the substrate. These energies, reported in Table I have been computed from the minimization of the potential energy by a conjugate gradient method.

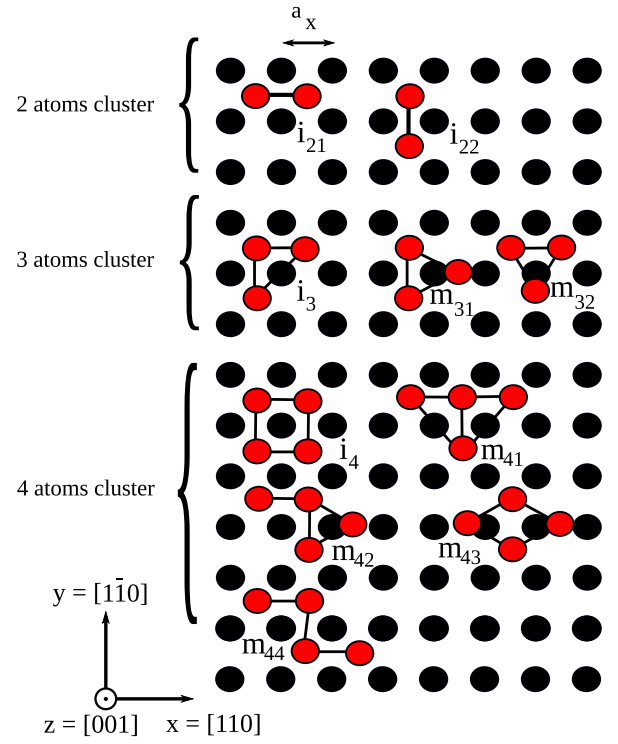

FIG. 3. Main observed conformations of clusters of two, three, and four atoms on the (001) surface of a fcc substrate. Red and black atoms, respectively, represent clusters and substrate atoms. For threeatom clusters, the different orientations of the clusters are labeled by numbers from 1 to 4 .

Below, we first show that the oscillation of the probability distributions $P_{i}(x)$ in the vicinity of antinodes of the transverse substrate displacement field (TSDF) can be explained by the occurrence of these stable and metastable conformations. We then address the interpretation of the probability distributions $P_{i}(x)$ in the vicinity of nodes of TSDF.

\section{Interpretation of the probability distributions $P_{i}(x)$ in the vicinity of antinodes of TSDF}

Two-atom clusters. The oscillations of $P_{2}(x)$ reported in Fig. 2(b) are attributed to the occurrence of two conformations $i_{21}$ and $i_{22}$ oriented, respectively, along the [110] and [11 0$]$ directions. Indeed, the projection of their centers of mass on the [110] direction lie, respectively, on and in-between [1110] atomic columns of the substrate. The distance $a_{x} / 2$, i.e., half the lattice substrate parameter between these two projections, agrees with the one between two successive maxima of the probability distribution $P_{2}(x)$. In addition, from Fig. 2(b),

TABLE I. Cluster energies of the most observed conformations of the clusters of two, three, and four atoms on the (001) surface calculated at $0 \mathrm{~K}$ in absence of any StSAW.

\begin{tabular}{lcc}
\hline \hline & Configurations & Energies \\
\hline Two-atom clusters & $i_{21}$ and $i_{22}$ & -10.95 \\
Three-atom clusters & $i_{3}$ & -17.97 \\
& $m_{31}$ or $m_{32}$ & -17.92 \\
Four-atom clusters & $i_{4}$ & -26.88 \\
& $m_{41}$ & -25.01 \\
& $m_{42}$ & -24.98 \\
& $m_{43}$ & -24.93 \\
& $m_{44}$ & -24.90 \\
\hline
\end{tabular}


the intensities of the maxima of $P_{2}(x)$ are more pronounced one maxima out of two in the vicinity of antinodes of the TSDF: the preferred cluster conformation is $i_{22}$ corresponding to the cluster lying along the [1 10$]$ direction. Indeed, in this conformation, the cluster is not sensitive to the strain field induced by the StSAW along the [110] direction, hence minimizing its average elastic energy.

Three-atom clusters. Figure 3 presents the most stable conformation referred as $i_{3}$ and two metastable conformations $m_{31}$ and $m_{32}$ of the three-atom clusters, responsible of the oscillations of $P_{3}(x)$. From the calculation of the minimum energies of both conformations at $0 \mathrm{~K}$ reported in Table I and using the Boltzmann factor, we deduce that the probability to observe conformations $i_{3}$ would be 1.24 higher than the one to observe $m_{31}$ or $m_{32}$ at the considered temperature $T=0.24$ in the absence of any StSAW.

From the examination of Fig. 2(c) in the vicinity of antinodes of the TSDF, and as clearly seen in the inset of Fig. 2(c), the maxima of $P_{3}(x)$ are formed by a double peak. Each doubled peak is spaced by $a_{x}$ while the two peaks inside a double peak are spaced by about $a_{x} / 3$. This probability distribution corresponds to the projection along the [110] direction of the centers of mass of the $i_{3}$ and $m_{31}$ conformations. Indeed, from both minimum energy conformations $i_{3}$ and $m_{31}$ evaluated at $0 \mathrm{~K}$ in the absence of any StSAW, we deduce (i) the center of masses of conformations $i_{3}$ and its mirror conformations [compared to the plane (110)] are spaced by $0.33 a_{x}$ along the [110] direction; (ii) the center of masses of conformation $i_{3}$ and $m_{31}$ are spaced by a distance $0.014 a_{x}$. Since this latter distance is very small compared to the lattice parameter, we believe that both conformations $i_{3}$ and $m_{31}$ (and their equivalent conformations obtained by symmetry) contribute to the observation of a double peak. Besides, the projection along the [110] direction of the centers of mass of the $m_{32}$ conformations [or its symmetric conformation compared to plane (110)] should also provide a peak lying on the substrate [110] atomic columns. This central peak is not observed in the inset of Fig. 2(c). Due to the presence of the StSAW inducing an average elastic energy depending on the cluster orientation, conformations $m_{32}$ are presumably less favorable than conformations $m_{31}$ or $i_{3}$; as a result, the $m_{32}$ conformation has no observable signature in the $P_{3}(x)$ curve.

Four-atom clusters. Figure 3 reports the most stable $i_{4}$ and the four first metastable $m_{4 i}(i \in\{1,2,3,4\})$ conformations (ordered by increasing energy) of the four-atom cluster. Figure 3 reports only plane conformations, but we have also investigated clusters with an atom on top of the three others: all three-dimensional investigated structures present less stable conformations than the one reported in Fig. 3. The stable $i_{4}$ and metastable $m_{4 i}(i \in\{1,2,3,4\})$ conformations have their center of mass, respectively, on and in-between substrate [1 10$]$ atomic columns. From the calculation of the minimum energies of these conformations at $0 \mathrm{~K}$ reported in Table I, using the Boltzmann factor and orientation degeneracy, the probability to observe conformations $i_{4}$ should be, respectively, 600, 340, 1677, and 950 higher than the one to observe $m_{41}, m_{42}$, $m_{43}$, and $m_{44}$ at the considered temperature $T=0.24$ in the absence of any StSAW [20]. From Fig. 2(d), in the vicinity of antinodes of the TSDF, the probability distribution $P_{4}(x)$ presents significant peaks spaced by $a_{x}$ corresponding to the
TABLE II. Structuring energy $\Delta E_{i}^{\text {eff }}$ as a function of the size $i$ of the cluster $i=\{1,2,3,4\}$.

\begin{tabular}{lcccc}
\hline \hline$i$ & 1 & 2 & 3 & 4 \\
\hline$\Delta E_{\mathrm{i}}^{\mathrm{eff}} / k_{B} T$ & 0,64 & 0,82 & 0,85 & 1,31 \\
\hline \hline
\end{tabular}

center-of-mass positions of the $i_{4}$ conformations. In-between these peaks, very tiny peaks are observed and correspond to the center-of-mass positions of the metastable conformations. Hence, the $i_{4}$ conformation is much more probable than the metastable one. However, the ratio of the probabilities to observe center-of-mass positions on (corresponding to conformation $i_{4}$ ) and between (corresponding to metastable) substrate [1 10 ] atomic columns is much smaller (about 30) than the one expected (few hundred) in the absence of StSAW, suggesting that average elastic energies of metastable conformations strongly affect the stability of the clusters in the presence of the StSAW.

\section{Interpretation of the probability distributions $P_{i}(x)$ in the vicinity of nodes of TSDF}

Finally, the interpretation of $P_{i}(x), i \in\{1,2,3,4\}$, in the vicinity of the nodes of the TSDF is difficult: indeed, nodes of the TSDF correspond to antinodes of the longitudinal displacement field [9]: the clusters [and even the adatom in Fig. 2(a)] are periodically displaced by this longitudinal field which consequently widens the maxima peak in the probability density curves. This widening is especially clearly observable on $P_{1}(x)$ and $P_{4}(x)$. The interpretation or prediction of the curve $P_{i}(x)$ then becomes a difficult problem out of the scope of this study [21].

\section{B. Self-organization intensity}

In order to quantify the self-organization intensity, we use an energy scale based on the Boltzman constant as already proposed in Ref. [6]. However, in order to take into account the spreading of the probability distributions peaks around stable positions of the clusters, we propose to use the following definition for the structuring energy $\Delta E_{i}^{\text {eff }}$ :

$$
\frac{\Delta E_{i}^{\mathrm{eff}}}{k_{B} T}=\ln \frac{\int_{x_{a}-a_{x} / 2}^{x_{x}+a_{2} / 2} \int_{y_{a}-a_{y} / 2}^{y_{a}+a_{y} / 2} p_{i}(x, y) d x d y}{\int_{x_{n}-a_{x} / 2}^{x_{n}+a_{x} / 2} \int_{y_{n}-a_{y} / 2}^{y_{n}+a_{y} / 2} p_{i}(x, y) d x d y},
$$

where $x_{a}, y_{a}\left(x_{n}, y_{n}\right)$ correspond to the stable position of the clusters at an antinode (node) of the TSDF. $a_{x}=a_{y}$ are the substrate lattice parameters along the [110] and [1 $\overline{1} 0]$ directions. This definition of the structuring energy [Eq. (2)] is more reliable than the definition previously proposed $[6,8]$ since it takes into account the full diversity of the cluster conformations. Table II reports the computed structuring energies $\Delta E_{i}^{\text {eff }}$ from Fig. 2 as a function of the cluster size.

The self-organization intensity is increasing with the cluster size. This result may be related to the diminution of the diffusion coefficient of the clusters with the size: a large cluster, once it reaches a low effective energy position at an antinode of the TSDF, has much less probability (on the simulation time scale) to change its position. Since, clusters behave as 
adatoms when submitted to a StSAW, we expect that the physical mechanism responsible of this self-organization is the same as the one operating on a single atom: the longitudinal displacement field induced a periodic motion of the cluster center of mass in the crystalline potential of the substrate. This motion results in an induced force at the pulsation of the StSAW: the average effect of this force drives the clusters and adatoms towards a node of the force corresponding also to an antinode of the TSDF [9]. In addition, although we do not investigate the effect of the temperature in this study, we can also expect that the self-organization intensity of clusters decreases with the temperature as it is the case for adatoms [9].

\section{SELF-ORGANIZATION DURING A GROWTH PROCESS}

From our previous studies and the above results, both adatoms and small clusters have the same physical behavior on a substrate submitted to a StSAW: they have an enhanced probability to be in the vicinity of an antinode of the TSDF. Therefore, during a growth process on a substrate submitted to a StSAW-like epitaxial molecular beam epitaxy, adatoms are expected to diffuse toward antinodes of the TSDF and to form small clusters in these regions. However, the probability to form a cluster in the vicinity of nodes of the TSDF will presumably not be zero. But, since both clusters and adatoms diffuse toward antinodes of the TSDF, small clusters will go and remain in these latter regions. Of course, only stable clusters the size of which exceeds the critical size will be stable and will be able to diffuse, others will dissociate [1,2,22]. The critical size depends on the deposition rate, temperature, and bond energies. Newly deposited adatoms will be trapped by these already existing stable clusters contributing to their growth. This scheme can operate provided there is a sufficiently low adatom depositing flux: a freshly deposited adatom should have the time to diffuse towards a small cluster before having the chance to nucleate a new cluster from the gathering with other adatoms. Similarly, a freshly formed cluster should have the time to diffuse towards the antinodes of the TSDF before its size (resulting of its growth) prevents its efficient diffusion. In other words, the diffusing length of adatoms and small clusters need to be of the order or larger than half the StSAW wavelength. The expected results of such a growth are the presence of clusters uniformly distributed on the antinodes of the TSDF. We below address the relevance of this growth scenario by testing it in MD simulations. All MD parameters are identical to the ones described in Sec. II except the $x$ size of the simulation cell that has been enlarged: $L_{x}=4 \lambda$. The growth is simulated by depositing 40 adatoms evenly distributed on the substrate surface every 8000 time units. Since two-atom clusters are stable on the simulation time scale within these conditions (see Sec. III), the critical size is expected to be one in these simulations (although we did not try to evaluate it precisely). Figures 4(a) and 4(b) report the position $x, y$ of deposited atoms after 32000 time units without and with the presence of the StSAW. Figure 4(c) reports the same quantity as Fig. 2(e). Whereas, without StSAW, clusters form at random $x$ position, clusters that have grown in the presence of the StSAW are all in the vicinity of the antinodes of the TSDF. The apparent elongated (in the $y$ direction shape)

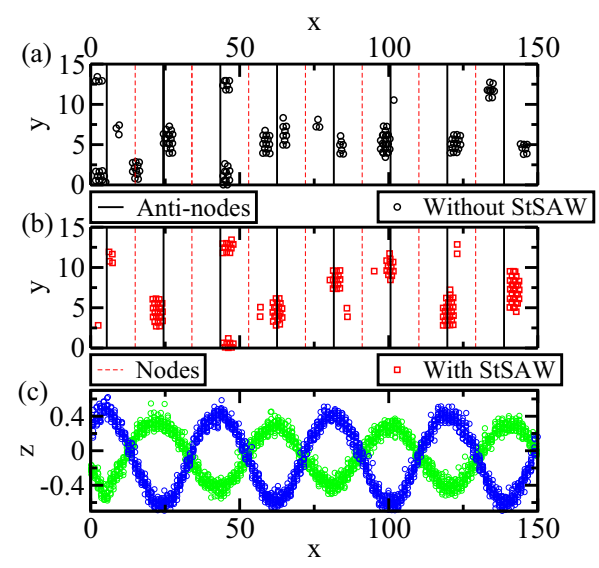

FIG. 4. (a) $x, y$ positions of adatoms deposited on the (001) surface of fcc substrate after 32000 time units and (b) on a substrate submitted to a StSAW propagating along the [110] at $T=0.24 .40$ atoms are deposited every 8000 time units. (c) Same as Fig. 2(e).

of clusters is due to the difference in axis scales in Fig. 4. The cluster aspect ratio is about unity. Clusters are crystalline and are mostly bidimensional (one monolayer thick) but some of them present very few atoms on the second layer. The biggest cluster is composed of 32 atoms. Due to the very few number of clusters, a size distribution calculated from Fig. 4 is not relevant. The main conclusion of Fig. 4 is to confirm that clusters containing up to tens of atoms self-organize on a substrate submitted to a StSAW. This simulation thus confirms the growth scenario proposed above.

\section{CONCLUSION}

The diffusion of clusters composed of few atoms and tens of atoms on a crystalline substrate submitted to a StSAW has been studied. The StSAW self-organizes the cluster diffusion. The probability to find a cluster in the vicinity of an antinode of the TSDFt is enhanced. The physical mechanism responsible for this self-organization is proposed to be the same as the one operating on a single atom: the longitudinal displacement field induced a periodic motion of the cluster center mass in the crystalline potential of the substrate. The detailed shapes of the probability distribution of mass-center positions can be explained for small clusters from the different possible cluster conformations and orientations on the substrate. A scenario on the effect of a StSAW during an epitaxial growth experiment has been proposed and its relevance evidenced. The StSAW is expected to drive the diffusion of the small clusters that will themselves act as attractive centers for newly deposited atoms. As a result, clusters will be spatially periodically located and spaced by half the StSAW wavelength. To go beyond our simple theoretical evidences, we hope that this work will motivate growth experiments involving StSAW. Propagative SAWs have been tentatively used in growth experiments in the 1990's [23,24]: although no noticeable results have arisen from these studies, they have evidenced that the epitaxial growth could be performed in the presence of a SAW. 
[1] J. Venables, Introduction to Surface and Thin Film Processes (Cambridge University Press, Cambridge, 2000).

[2] A. Pimpinelli and J. Villain, Physics of Crystal Growth (Cambridge University Press, Cambridge, 1998).

[3] H. Brune, M. Giovannini, K. Bromann, and K. Kern, Nature (London) 394, 451 (1998).

[4] A. Turala, P. Regreny, P. Rojo-Romeo, and M. Gendry, Appl. Phys. Lett. 94, 051109 (2009).

[5] R. A. Segalman, A. Hexemer, and E. J. Kramer, Phys. Rev. Lett. 91, 196101 (2003).

[6] C. Taillan, N. Combe, and J. Morillo, Phys. Rev. Lett. 106, 076102 (2011).

[7] N. Combe, C. Taillan, and J. Morillo, Phys. Rev. B 85, 155420 (2012).

[8] C. Taillan, N. Combe, and J. Morillo, Phys. Rev. B 85, 155421 (2012).

[9] C. Taillan, N. Combe, and J. Morillo, Eur. J. Phys. B 88, 317 (2015).

[10] C. Wu, V. Y. Zaitsev, and L. V. Zhigilei, J. Phys. Chem. C 117, 9252 (2013).

[11] M. V. Shugaev, A. J. Manzo, C. Wu, V. Y. Zaitsev, H. Helvajian, and L. V. Zhigilei, Phys. Rev. B 91, 235450 (2015).
[12] S. J. Plimpton, J. Comput. Phys. 117, 1 (1995).

[13] P. Guan, D. R. Mckenzie, and B. A. Pailthorpe, J. Phys.: Condens. Matter 8, 8753 (1996).

[14] G. L. Kellogg and A. F. Voter, Phys. Rev. Lett. 67, 622 (1991).

[15] M. Zinke-Allmang, L. C. Feldman, and M. H. Grabow, Surf. Sci. Rep. 16, 377 (1992).

[16] J. M. Wen, S. L. Chang, J. W. Burnett, J. W. Evans, and P. A. Thiel, Phys. Rev. Lett. 73, 2591 (1994).

[17] H. Brune, Surf. Sci. Rep. 31, 125 (1998).

[18] C. M. Chang, C. M. Wei, and S. P. Chen, Phys. Rev. Lett. 85, 1044 (2000).

[19] J. Yang, W. Hu, J. Tang, and M. Xu, J. Phys. Chem. C 112, 2074 (2008).

[20] This estimation neglects the vibrational entropy but take into account the configurational entropy .

[21] P. Jung, Phys. Rep. 234, 175 (1993).

[22] J. G. Amar and F. Family, Phys. Rev. Lett. 74, 2066 (1995).

[23] M. Takahashi, S. Sakakibara, M. Tsunoda, H. Shoji, and T. Wakiyama, J. Magn. Magn. Mater. 126, 62 (1993).

[24] M. Takahashi, H. Shoji, and M. Tsunoda, J. Magn. Magn. Mater. 134, 403 (1994). 